\title{
INTRACRANIAL INFECTION RATE; \\ POST OPERATIVE PREVALENCE AT CIVIL HOSPITAL KARACHI, PAKISTAN.
}

1. Resident Medical Officer Department of Neurosurgery Civil Hospital Karachi.

2. Assistant Professor Department of Neurosurgery Civil Hospital Karachi.

3. Assistant Professor Department of Neurosurgery Civil Hospital Karachi.

4. Resident Officer Department of Neurosurgery Civil Hospital Karachi.

5. Associate Professor Department of Neurosurgery Civil Hospital Karachi.

6. Profesor and Head of Department, Department of Neurosurgery Civil Hospital Karachi.

Corresponence Address: Dr. Qazi Muhammad Zeeshan Resident Medical Officer

Department of Neurosurgery Civil Hospital Karachi.

Address: Flat No: A 315 Billys Tower Main Rashid Minhas Road Karachi. doctor.karachi@gmail.com

Article received on:

06/06/2016

Accepted for publication: $30 / 11 / 2016$

Received after proof reading: $14 / 02 / 2017$

\section{INTRODUCTION}

One of the serious complications of intracranial surgery is infection, and it requires immediate intervention, it presents as meningitis, sub dural empyema or cerebral abscess. $6,9,14,17,21$

Meningitis commonly occurs after surgical procedures which have an approach from the posterior fossa and is frequently found to be associated with a CSF leak, it is diagnosed via lumbar puncture and subsequent CSF analysis and treated with a vigorous course of antibiotics and steroids. ${ }^{3,13,16}$ Other cases of infection such as subdural empyema, abscess and flap infection required operation with removal of the offending agent. $6,9,11,12,14,21$ The surgical procedure then done is to remove the debris and allow the antibiotics to work effectively and efficiently. In a large series of procedures the rate of infection after prophylactic antibiotics was found to be 0.8 to $7 \% .^{1,5,9,15,19}$ Some studies suggest an even higher rate of infection greater than $10 \% .4,5,10,18,20,22,23,24$ These included cases of both surgical and medical causes and also cases which were not operated, we underwent the review in our centre of the last ten years, and focused more on the infections that were observed after surgical procedures, excluding those that underwent simple wound revision and evacuation of pus.

\section{MATERIALS AND METHODS}

The type of study is a retrospective analysis of cases of craniotomy, studying the files of patients who had the procedure done at the department over the last ten years period (2005 to 2015) at Civil Hospital Karachi Pakistan. To determine the 
incidence and factors associated with infection a sub group analysis was done in all those patients in whom the intracranial infection was caused by a cranial surgery. The incidence, associated factors and infectious organism was also noted. Patients who were administered antibiotics for the treatment of infection (without any surgical intervention, as in cases of meningitis and cellulitis) were not included also those who were treated for wound infection without intracranial involvement. Cases of patients having cerebral abscess, subdural abscess, epidural abscess, infection of the bone flap were included. The patients included in the study came to the department for surgical procedures either as emergency or elective cases, patients who had a prior surgical procedure done at some other institute were excluded from the study. Prophylactic antibiotic was administered to all the patients as $1 \mathrm{gm}$ of third generation cephalosporin one hour before the incision, and was followed in most cases by a one day course of postoperative antibiotics, but some cases were those whom did not receive post operative antibiotics. After shaving the site of incision, the area was scrubbed with pyodine solution, and the required procedure was performed. Data was analyzed using SPSS version 20 , chi square test was used to analyze the difference between the groups and non parametric Mann Whitney $U$ test was utilized where one of the groups contained continuous variable data.

\section{RESULTS}

In the period of study that is from the year 2005 to 2015 a total of 5800 cranial surgical procedures were performed by a team of 30 neurosurgeons and residents. And a total $n=116(2 \%)$ procedures were done due to post operative infection on a total of $n=70$ patients. $N=37$ patients were male and $n=33$ patients were female, the median age of patients was 50 years and the age range was from 2 to 75 years. The procedures were performed as elective procedures in $n=56$ (80\%) patients and as an emergency case in $n=14$ (20\%) patients. The most common complaint in the patient group was a change in mental status which was reported in $n=25(35.71 \%)$ of patients. Other presenting complaints are listed in Table-l.
The majority of patients had more than 1 type of infection, and the most common type of infection found in the patient group was an epidural empyema found in $n=38(54.28 \%)$ patients, the other types of infections were found as subdural empyema in $n=10$ (14.28\%) patients, cerebral abscess and abscess of the posterior fossa in $n=$ $11(15.71 \%)$ and $n=3(4.28 \%)$ patients, wound infection was found in $n=36(51.42 \%)$ patients and $n=31$ (44.28\%) patients had an infected bone flap all of these infections required redoing of the surgery, and significant association was not found between bone flap removal and the need for multiple procedures for the purposes of curbing the infectious agent a $p$ value of less than 0.318 was obtained via a chi square test. The mean number of operations that were performed was 1.65 (median $=1$, and a range between 1 and 5) $n=40(57.14 \%)$ of patients had only one surgical procedure done to remove the infectious debris, while $n=13(18.57 \%)$ had two procedures, and $\mathrm{n}=7(10 \%)$ had three surgical procedures done for successful treatment. In $n=2(2.85 \%)$ patients 4 procedures had to be done. The most common organism that was found to be the cause of infection was methicillin sensitive Staphylococcus aureus, refer to Table-I. No significant association was found between the type of organism and the number of surgical interventions needed the $p$ value for a negative culture was found to be less than 0.168 , and $p$ value for multiple infectious agents was found to be less than 0.091 and the $\mathrm{p}$ value for staphylococcus spp was found to be less than 0.548 , according to the chi square tests done. The surgical procedure which had highest infection rate was craniotomy as done for tumor or mass lesion seen in $n=32(45.71 \%)$ patients, for rest of the frequencies refer to TableII. The duration for craniotomy and presentation with infection was at a median of 1.5 months with a range of 4 days to 1 year duration. For $n=37(52.85 \%)$ of patients they presented with infection after their first operation and for $n=20$ (28.57\%) of patients presented with infection after their second procedure. And for $n=13$ (18.57\%) had more than two procedures done before development of infection. 


\begin{tabular}{|l|c|}
\hline \multicolumn{1}{|c|}{ Infection } & Number of patients \\
\hline Subdural empyema & $10(14.28 \%)$ \\
\hline Epidural Empyema & $38(54.28 \%)$ \\
\hline Cerebral abscess & $11(15.71 \%)$ \\
\hline $\begin{array}{l}\text { Abscess of the posterior } \\
\text { fossa }\end{array}$ & $3(4.28 \%)$ \\
\hline Infection of the bone flap & $31(44.28 \%)$ \\
\hline Wound infection & $36(51.42 \%)$ \\
\hline Symptoms present & \\
\hline Purulent discharge & $24(34.28 \%)$ \\
\hline Change in the mental & $25(35.71 \%)$ \\
\hline status & $14(20 \%)$ \\
\hline Headache & $10(14.28 \%)$ \\
\hline Swelling & $15(21.42 \%)$ \\
\hline Fever & $3(4.28 \%)$ \\
\hline Seizure & \\
\hline & \\
\hline Type of Organism & $3(4.28 \%)$ \\
\hline Staphylococcus spp. & $14(20 \%)$ \\
\hline Methicillin resistant & $8(11.42 \%)$ \\
\hline Methicillin sensitive & $3(4.28 \%)$ \\
\hline Coagulase-negative & $3(4.28 \%)$ \\
\hline Steptococcus spp & $7(10 \%)$ \\
\hline Propionibactrium spp & $4(5.71 \%)$ \\
\hline Multiple organisms & $6(8.57 \%)$ \\
\hline Enterobacter & $3(4.28 \%)$ \\
\hline Pseudomonas & $8(11.42 \%)$ \\
\hline Serratia & $3(4.28 \%)$ \\
\hline Other & $8(11.42 \%)$ \\
\hline Candida spp & \\
\hline Culture negative & \\
\hline Table-l. Type of infection in patients and the most \\
\hline
\end{tabular}

\section{DISCUSSION}

In a large series of neurosurgical procedures the incidence of infections was found to be from 0.8 to $7 \%$ (despite prophylactic antibiotics, and including non operative cases). 1,5,9,15,19 In our study we reviewed our experience at our department which is one of the highest volume centre in Karachi Pakistan. We conducted a retrospective review in order to find out the rate of infection that required surgical intervention, and only focused on patients who required reoperation for removal of debris or pus evacuation. We found a total of $n=70$ (total number of procedures= 116) patients out of the $n=5800$ patients that were operated upon in the ten year period, for an incidence of $2 \%$, which is lower than some other series that report greater than 1000 procedures. According to McClelland and Hall they report the postoperative incidence of infection to be $0.63 \%$ in a total of 1587 elective procedures, which an incidence rate similar to our study. $35 \%$ of their cases of infection were associated with indwelling catheters, where as this was much lower in our study. In our study the most common organism was staph aureus spp which occurred in $35.71 \%$ of cases, which is similar to other studies which report S. aureus in upto $50 \%$ of cases. ${ }^{2,7,8,9,20}$ In a study by Korinek et al, he studies a series of 2944 patients who had craniotomy done, and there were $3 \%(n=87)$ occurrences of post operative infection, excluding $n=56$ meningitis, and $n=30$ cases of wound infection. ${ }^{7}$ The risk factors he identified for infection were leakage of CSF, and subsequent surgical procedures. Other risk factors included, emergency procedures, clean contaminated or dirty wounds, an operative time of greater than 4 hours duration, while prophylactic antibiotic was not a determining factor for infection. ${ }^{7}$ But in our study we were not able to find a statistically significant increase in infection for emergency versus elective case. The

\begin{tabular}{|l|c|c|c|}
\hline \multicolumn{1}{|c|}{ Type of procedure } & Emergency procedure & Elective procedure & Total \\
\hline Craniotomy (tumor) & $0(0 \%)$ & $32(45.71 \%)$ & $32(45.71 \%)$ \\
\hline Craniotomy (hemorrhage) & $1(1.42 \%)$ & $1(1.42 \%)$ & $2(2.85 \%)$ \\
\hline Craniotomy (vascular lesion) & $0(0 \%)$ & $10(14.28 \%)$ & $10(14.28 \%)$ \\
\hline Craniectomy (decompression) & $0(0 \%)$ & $2(2.85 \%)$ & $2(2.85 \%)$ \\
\hline Infratentorial craniectomy & $1(1.42 \%)$ & $1(1.42 \%)$ & $2(2.85 \%)$ \\
\hline Surgery for seizures & $0(0 \%)$ & $1(1.42 \%)$ & $1(1.42 \%)$ \\
\hline$\quad$ Trauma management & & & \\
\hline Craniotomy (hemorrhage) & $8(11.42 \%)$ & $1(1.42 \%)$ & $9(12.85 \%)$ \\
\hline Craniectomy (decompression) & $4(5.71 \%)$ & $0(0 \%)$ & $4(5.71 \%)$ \\
\hline Other & $0(0 \%)$ & $8(11.42 \%)$ & $8(11.42 \%)$ \\
\hline
\end{tabular}

Table-II. Types of surgical procedures. 
retrospective case series analysis reflects the rarity of the infection, and it helps in defining its natural history and progression. We did not find any statistical significance when it come to the type of organism and number of surgical procedures done, which may be due to the small number of patients in the series. One of the cofounding factor is the multiple number of surgeons who were involved in the procedures, we did not compare the time duration of the procedure to the incidence of infection, but previous studies have shown that there exists a correlation. ${ }^{7}$ It is quite possible that many patients were not included because they had gone to some other facility for treatment of infection. A total of $n=31$ (44.28\%) patients had bone flap removal at surgery for the infection, which was due to the observation that the bone flap was not infected, that is in a case of deep abscess, there is no statistically significant association found between bone flap removal and infection occurring again, having a $p$ value of less than 0.318 .

\section{CONCLUSION}

The postoperative infection is one of the most important complication of cranial surgical procedures and required immediate attention and treatment, even after taking all the precautionary measures to ensure sterility a small number of patients still develop severe infection needing reoperation for removal of debris and pus.

Copyright@ 30 Nov, 2016.

\section{REFERENCES}

1. Alazia $M$, Bruder $N$ : [Antibiotic prophylaxis in craniocerebral wounds.] Ann Fr Anesth Reanim 11:705-710, 1992 (Fr).

2. Apisarnthanarak $A$, Jones $M$, Waterman $B M$, Carroll $C M$, Bernardi R, Fraser VJ: Risk factors for spinal surgicalsite infections in a community hospital: a casecontrol study. Infect Control Hosp Epidemiol 24:31-36, 2003.

3. Benca J, OndrusovaA, Kisac P, Krcmery V: Postoperative meningitis: shift in etiology? Neuro Endocrinol Lett 28 (3 Suppl):22, 2007.

4. Djindjian M, Lepresle E, Homs JB: Antibiotic prophylaxis during prolonged clean neurosurgery. Results of a randomized doubleblind study using oxacillin. J Neurosurg 73:383-386, 1990.
5. Gaillard T, Gilsbach JM: Intra-operative antibiotic prophylaxis in neurosurgery. A prospective, randomized, controlled study on cefotiam. Acta Neurochir (Wien) 113:103-109, 1991.

6. Hlavin ML, Kaminski HJ, Fenstermaker RA, White RJ: Intracranial suppuration: a modern decade of postoperative subdural empyema and epidural abscess. Neurosurgery 34:974-981, 1994.

7. Korinek AM: Risk factors for neurosurgical site infections after craniotomy: a prospective multicenter study of 2944 patients. The French Study Group of Neurosurgical Infections, the SEHP, and the C-CLIN Paris-Nord. Service Epidémiologie Hygiène et Prévention. Neurosurgery 41:1073-1081, 1997.

8. Korinek AM, Baugnon T, Golmard JL, van Effenterre $\mathrm{R}$, Coriat P, Puybasset L: Risk factors for adult nosocomial meningitis after craniotomy: role of antibiotic prophylaxis. Neurosurgery 59: 126-133, 2006.

9. McClelland S III, Hall WA: Postoperative central nervous system infection: incidence and associated factors in $\mathbf{2 1 1 1}$ neurosurgical procedures. Clin Infect Dis 45:55-59, 2007.

10. Mindermann T, Zimmerli W, Gratzl O: Randomized placebo-controlled trial of single-dose antibiotic prophylaxis with fusidic acid in neurosurgery. Acta Neurochir (Wien) 121:9-11, 1993.

11. Nathoo N, Nadvi SS, van Dellen JR: Cranial extradural empyema in the era of computed tomography: a review of 82 cases. Neurosurgery 44:748-754, 1999.

12. Nathoo N, Nadvi SS, van Dellen JR, Gouws E: Intracranial subdural empyemas in the era of computed tomography: a review of 699 cases. Neurosurgery 44:529-536, 1999.

13. Nazzaro JM, Craven DE: Successful treatment of postoperative meningitis due to Haemophilus influenzae without removal of an expanded polytetrafluoroethylene dural graft. Clin Infect Dis 26: 516-518, 1998.

14. Post EM, Modesti LM: "Subacute" postoperative subdural empyema. J Neurosurg 55:761-765, 1981

15. Rocca B, Mallet MN, Scemama F, Malca S, Chevalier $A$, Gouin F: [Perioperative remote infections in neurosurgery. Role of antibiotic prophylaxis.] Presse Med 21:2037-2040, 1992 (Fr).

16. Rousseau JM, Soullie B, Villevielle T, Koeck JL: Efficiency of cefepime in postoperative meningitis attributable to Enterobacter aerogenes. J Trauma 
50:971, 2001.

17. Rousseaux M, Lesoin F, Clarisse J, Lozes G, Jomin M: [Postoperative abscesses and empyemas. Apropos of 13 cases.] Neurochirurgie 32:304-310, 1986 (Fr).

18. Shapiro M, Wald U, Simchen E, Pomeranz S, Zagzag D, Michowiz SD, et al: Randomized clinical trial of intraoperative antimicrobial prophylaxis of infection after neurosurgical procedures. J Hosp Infect 8:283-295, 1986.

19. Tenney JH, Vlahov D, Salcman M, Ducker TB: Wide variation in risk of wound infection following clean neurosurgery. Implications for perioperative antibiotic prophylaxis. J Neurosurg 62: 243-247, 1985.

20. van Ek B, Bakker FP, van Dulken $H$, Dijkmans BA: Infections after craniotomy: a retrospective study. $J$ Infect 12:105-109, 1986.

21. Vogelsang JP, Wehe A, Markakis E: Postoperative intracranial abscess-clinical aspects in the differential diagnosis to early recurrence of malignant glioma. Clin Neurol Neurosurg 100: 11-14, 1998.

22. Winkler D, Rehn H, Freckmann N, Nowak G, Herrmann HD: Clinical efficacy of perioperative antimicrobial prophylaxis in neurosurgery-a prospective randomized study involving 159 patients. Chemotherapy 35:304-312, 1989.

23. Young RF, Lawner PM: Perioperative antibiotic prophylaxis for prevention of postoperative neurosurgical infections. A randomized clinical trial. J Neurosurg 66:701-705, 1987.

24. Zhu XL, Wong WK, Yeung WM, Mo P, Tsang CS, Pang $\mathrm{KH}$, etal: $A$ randomized, double-blind comparison of ampicillin/sulbactam and ceftriaxone in the prevention of surgical-site infections after neurosurgery. Clin Ther 23:1281-1291, 2001.

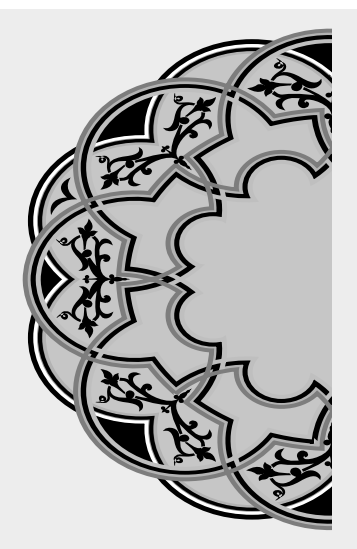

\title{
"Health is not valued till sickness comes."
}

\author{
Thomas Fuller
}

AUTHORSHIP AND CONTRIBUTION DECLARATION

\begin{tabular}{|c|c|c|c|}
\hline Sr. \# & Author-s Full Name & Contribution to the paper & Author $=\mathbf{s}$ Signature \\
\hline 1 & Dr. Qazi M. Zeeshan & Concept, Layout & 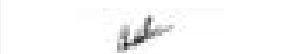 \\
\hline 2 & Dr. Muhammad Faiq Ali & Write up, Drafting & $\operatorname{mes}$ \\
\hline 3 & Dr. Shiraz Ahmed Gauri & Write up Data analysis & rend \\
\hline 4 & Dr. Syed Muneeb Younus & Data Collection Data entry & ind \\
\hline 5 & Dr. Muhammad Imran & $\begin{array}{l}\text { Statistical analysis, Final } \\
\text { layout }\end{array}$ & $y=$ \\
\hline 6 & Prof. Dr. Junaid Ashraf & Final layout, Analysis & \\
\hline
\end{tabular}

